\title{
A perceptual-confusion account of the WSE in the target search paradigm
}

\author{
KENNETH R. PAAP and SANDRA L. NEWSOME \\ New Mexico State University, Las Cruces, New Mexico 88003
}

\begin{abstract}
Subjects were required to search through four-letter displays for one member from the target set $M, N, P$, or R. The displays were words, pseudowords, or consonant strings. The results show that significant word superiority effects occur when target location is unknown, but that a location precue can eliminate these benefits of lexical context. Analysis of the types of errors that occur with and without a location precue indicates that the precue reduces the likelihood that the context letters of nonwords will be confused with the incorrect targets. These findings are consistent with a perceptual-confusion model that predicts that the context letters of words will be perceived more accurately than those of nonwords.
\end{abstract}

Letter recognition is usually better when a target is presented in a word than when it is presented either alone or in a nonword. Two paradigms have been used to study the effects of lexical context on letter recognition. The paradigm originally developed by Reicher (1969) requires subjects to choose between two alternatives that are presented after stimulus presentation. Since both the identity of the alternatives and the location of the critical letter vary from trial to trial and are not revealed until the onset of the masking field, we refer to this as the postcue procedure. In contrast, Bjork and Estes (1973) and Massaro (1973) have introduced a procedure wherein subjects search for the same small set of target alternatives on every trial of the experiment. We refer to this as the predesignated targets paradigm. Bjork and Estes used predesignated targets to reduce the shortterm memory requirements of the task, whereas Massaro was primarily concerned with controlling for the effects of orthographic redundancy at perceptual, as well as conceptual, levels of processing. Our experiments reveal another important difference between the paradigms, viz, that subjects searching for a well-learned set of targets will heavily bias the letter-recognition process toward the target set. The consequences of this are that the visual similarity between the context letters and the target letters, together with the amount of positional uncertainty concerning the target location, will critically determine the magnitude of the word advantages obtained with predesignated targets. Specifically, the data support

Portions of this research were presented at the meeting of the Rocky Mountain Psychological Association, Denver, April 1978. We thank Kelly Rudy and Jim McDonald for their contributions to various stages of this project. Earlier versions of this paper benefited from the comments of Dominic Massaro and the CIP group at NMSU. Requests for reprints should be sent to Kenneth R. Paap, Department of Psychology, Box 3452, New Mexico State University, Las Cruces, New Mexico 88003. the view that benefits of lexical context will accrue only when target location is unknown and primarily for displays whose context letters are highly confusable with the incorrect target alternatives. These results follow from the perceptual-confusion model that was developed by Paap, Newsome, and Rudy (Note 1) to specify the conditions that should produce word superiority effects (WSE) when predesignated targets are used.

The perceptual-confusion model starts with the premise, originally advanced by Smith (1971), that letter sequences corresponding to whole words can be perceptually synthesized on the basis of fewer letter features than would be necessary to perceive the constituent letters presented in isolation. If whole words can be perceived on the basis of fewer criterial features, then subjects will have more accurate information about the letters in a word than about the letters in a nonword. The importance of perceptual confusions in the predesignated-targets paradigm arises from the further assumption that the advantages of a word match should be much greater for the recognition of the context letters than for the target letter. The logic of this assumption is as follows. When the subject searches on every trial for the same small set of targets, it is likely that the letter units corresponding to the targets will have their criteria lowered to the point where they may be activated on the basis of very little evidence. This means that there is a very good chance that the input from the target location will activate the corresponding letter unit. However, lowering the criterion for the target units will also increase the probability that the input from a context letter will activate one of the incorrect target units. Assuming that word matches override incompatible or ambiguous matches at the level of individual letter units, it, therefore, follows that the occurrence of a word match is more likely to correct the errant matching of context-letter input. This, in turn, makes it 
less likely that a context letter will be mistaken for a target when the target is embedded in a word rather than a nonword.

As an example, consider an experiment that uses the predesignated target set $\mathrm{M}, \mathrm{N}, \mathrm{P}$, and $\mathrm{R}$ and a trial where the word NICK is presented. If the subject knows that the target will occur in the first position, then the decision will be based only on the evidence concerning the identity of the character located in the specified target location. Since $\mathbf{N}$ is a member of the target set, the $\mathrm{N}$ letter unit has already been primed and it is unlikely that the context letters could further enhance the chances of an $\mathrm{N}$ becoming synthesized in the first position. Accordingly, performance should be the same as in the nonword NJCK, where, once again, the recognition process must find the best match between the visual features detected in the first position and the four potential targets.

The situation is quite different when the target can occur in any position. In this case, the input from each location must be considered as a potential target and, occasionally, the input from a context letter may look just as much (or more) like one of the incorrect targets than the input from the target location looks like itself. For example, suppose that when NICK is presented, N, I, and C are unambiguously synthesized in the first three positions, but that the partial information extracted from the last position supports either K or R. (Support for an incorrect target, $R$ in this case, should occur often, since the model assumes that predesignating the targets leads to a reduction in the number of criterial features necessary to activate those letter units.) Since NICR is a nonword, the only potential word match is NICK. If the word NICK is activated, the evidence from the word match will support $\mathrm{N}$ in the initial position, and the subject will correctly respond that $\mathrm{N}$ was the target. In contrast, when a nonword such as NJCK is presented, the context NJC_ does not form a word with either $K$ or $R$ and either letter could be synthesized. When $R$ is synthesized, the subject will have to choose between the $\mathrm{N}$ seen in the first position and the $R$ seen in the last position. On these trials, the subject will have only a $50 \%$ chance of being correct. Performance will be lower with nonwords than with words, to the extent that this type of confusion occurs more often with nonwords.

Several predictions follow from the preceding analysis. First, under conditions of positional uncertainty, targets embedded in words should be recognized better than those presented in nonwords. Second, if target location is precued and subjects can consider only information about the target, there should be no differences between context types.

The final predictions concern the types of errors that subjects should make when target location is unknown. One type of error is based on context-letter input. That is, subjects will sometimes respond incorrectly because one of the context letters looks like one of the incorrect targets. The perceptual-confusion model predicts that this type of error will occur more often with nonwords than with words, since lexical access will frequently correct an incompatible and incorrect match that has occurred at the level of individual letter units. A second class of errors is based on target-letter input. That is, subjects will sometimes base their decisions on the correct input, but will synthesize the wrong target. Since the perceptualconfusion model assumes that lexical access will only occasionally lead to the correction of an incompatible and incorrect match of the target-letter input, the advantages of words over nonwords should be severely reduced on those trials where the subject bases his decision on target-letter input. In summary, errors based on context-letter input should show significant word advantages, whereas errors based on target-letter input should show significantly less, or even no, word advantage.

A reasonable index of the frequency of these two types of errors would be the distribution of errors that were similar vs. dissimilar to the target presented. If we again consider the target set $\mathrm{M}, \mathrm{N}, \mathrm{P}$, and $R$, the word BOMB provides an excellent example of this index. If the subject responds on the basis of information from the target location, errors should be similar to the target $\mathbf{M}$. That is, $\mathbf{N}$ should be chosen more often than $\mathbf{P}$ or $\mathbf{R}$. If the subject responds on the basis of information from one of the context letters, he would be likely to have synthesized one of the curved targets P or R. Particularly, it is quite likely that the partial information about either of the Bs may lead to the synthesis of a percept that looks just as $R$-like as the $M$ appears $\mathrm{M}$-like. Accordingly, the number of dissimilar errors provides a rough estimate of the number of errors made in response to context-letter input. Therefore, it follows that any advantages of location cuing should be nested almost completely in the decrease of dissimilar errors.

Obviously, the similar/dissimilar error index is susceptible to a great deal of noise. When the subject has very poor information about both the target and the context letters, he will simply guess. However, this should only work against our hypothesis that lexical constraint will greatly effect the distribution of dissimilar errors, but only marginally affect the number of similar errors. That is, if, for reasons extraneous to the perceptual-confusion model, subjects are in the guess state more often for nonwords than for words, then word advantages should be observed for both types of errors. The analysis of similar and dissimilar errors will be used to support the view that word advantages primarily occur because lexical context reduces the likelihood that a context letter will 
be confused with one of the incorrect targets. A second test of this hypothesis will examine the Location Cuing by Context Type interaction for displays with either high or low levels of confusability.

\section{EXPERIMENT 1}

The first experiment tests the predictions described above. All subjects searched for the predesignated targets $M, N, P$, or $R$ on every trial of the experiment. On half the trials, subjects were told in advance the location of the target letter, whereas they were forced to consider all four letter locations on the remaining trials. The effectiveness of a precue for target location is, of course, contingent upon the subject's ability to use the cue to segregate information about the target letter from that of the context letters. This segregation process on cued trials was maximized by shifting the fixation point from its normal central position to a position directly aligned with the upcoming target letter.

\section{Method}

Apparatus and Subjects. Three fields of an Iconix No. 6137 programmable tachistoscope were used to present the fixation point, test displays, and mask. The lamps in the tachistoscope are driven by an Iconix No. 6192-4 lamp driver with rise and fall times less than $.2 \mathrm{msec}$. Subjects placed their foreheads against a padded rest located just above the viewing window. Thirteen introductory psychology students plus the investigator S.N. served as subjects.

Stimulus materials. All test displays were composed of four letters that were presented for $30 \mathrm{msec}$. The upper case of the IBM Orator typing element was used to type each test display. Since the Orator type font is slightly larger than most pica styles, an elite space separated each of the letters in a display. This gives the large Orator letters the appearance of natural spacing. At the viewing distance used $(88 \mathrm{~cm})$, the width of a character subtended approximately $.12 \mathrm{deg}$ and the intercharacter space was about $.18 \mathrm{deg}$ of visual angle. Thus, the width of the entire display was about $1.02 \mathrm{deg}$. The mask consisted of a string of overlapping Os and Xs spatially coincident with the location of the test letters.

For all context types, the target letters were $\mathbf{M}, \mathbf{N}, \mathbf{P}$, and $\mathbf{R}$. The context in which the target appeared was of three types: words, pseudowords, and consonant strings. There were 20 words for each target, yielding a master set of 80 words. The position of the target letter was equally distributed across the four letter locations. Thus, there were five words for each combination of target letter and display location. The words for each quintet were selected from the Thorndike-Lorge (1944) count so that there would be one token from each of five frequency classes: $1,2,5,14$, and 23 per million. For example, the five words containing the target $R$ in the first position were RUSE, RAZE, RIFT, RIOT, and RUST. The pseudowords were formed by randomly replacing vowels in each word with other vowels. This yielded the strings RASU, REZO, RUFT, REIT, and ROST for the words listed above. Similarly, the consonant strings were formed by randomly replacing the vowels in each word with consonants, for example, RQSW, RTZD, RBFT, RKHT, and RGST. Hence, there were 240 different test displays in all.

Procedure. Each trial began with the subject being informed about the possible location of the target letter. On cued trials, the command one, two, three, or four was used to designate the serial position of the target letter in the upcoming display. On no-cue trials, the subjects were simply told no cue. Shortly after the verbal signal, the fixation point appeared for a full second. The fixation point was vertically positioned so that it was always just below the test display. The horizontal position of the fixation point was varied. On no-cue trials, it was perfectly centered. On cued trials, the fixation point was adjusted so that subjects would be fixating directly beneath the target letter. The subjects were told at the beginning of the experiment that they would always be looking directly at the target letter on a cued trial. The test display was followed by a mask that remained in view for $3 \mathrm{sec}$. The subjects responded verbally with one of the four target alternatives. Although no effort was made to speed the responses, most subjects began responding well before the termination of the mask.

The asynchrony in onset (SOA) of the test display and mask was adjusted during a training session of 48 to 96 trials so that each subject would have an overall error rate in the range of .25 to .50 . This insured that there would be at least a full quartile range before performance in particular conditions would be either perfect or at chance level. The SOAs ranged from 30 to $140 \mathrm{msec}$, with a median of $70 \mathrm{msec}$. Feedback was provided during the training session, but not during the experimental sessions.

The 240 different test displays were presented in the same random order in each of two experimental sessions. A coin toss was used to assign each trial of the first session to either the cued or the no-cue condition. In the second session, the cuing condition of each display was the reverse of its assignment in the first session.

\section{Results}

An analysis of variance was performed on the error rates, treating cuing (cue, no cue), context type (word, pseudoword, consonants), and target location $(1,2,3,4)$ as separate factors. The main effects of cuing $[F(1,13)=7.40, p<.05]$, context type $[F(2,26)=$ $8.01, \mathrm{p}<.01]$, and target location $[\mathrm{F}(3,39)=9.07$, $\mathrm{p}<.01]$, and the Cuing by Context Type interaction $[F(2,26)=3,60, p<.05]$ were significant. The main effect of target location reflects a $10 \%$ advantage of displays with targets in the first position over displays with targets in the other three positions. However, since target location failed to interact with the other variables of interest, the data were collapsed across target location in all further analyses.

The upper panel of Table 1 shows the average percentage of errors obtained with the three context types for each of the two conditions of cuing. Clearly, the benefits of cuing are highly dependent upon context type. There was virtually no effect of cuing on word trials, whereas the location cue improved performance for both nonword contexts by about $7 \%$.

No-cue condition. The first major prediction was that under conditions of positional uncertainty lexical context should facilitate recognition of the target letters. This prediction is clearly supported by the $10 \%$ advantage of words over consonant strings. Eleven of the 14 subjects showed this advantage, and a Wilcoxon signed-ranks test (Bradley, 1976) ${ }^{1}$ indicates that the differences in these two treatment effects is highly significant $[\mathrm{W}+(\min )=92, \mathrm{p}<.01]$. However, the advantage of pseudowords over consonant strings was only $2.3 \%$. Although 10 of the 14 subjects did better with the pseudowords, a Wilcoxon test failed to show a significant difference between 
Table 1

Mean Percentage of Errors for Each Context Type and the Type of Location Information

\begin{tabular}{|c|c|c|c|}
\hline & \multicolumn{3}{|c|}{ Context Type } \\
\hline & Words & Pseudowords & Consonants \\
\hline & & Experiment 1 & \\
\hline \multicolumn{4}{|l|}{ Cuing* } \\
\hline Cued & 36.8 & 38.2 & 39.8 \\
\hline \multirow[t]{2}{*}{ No Cue } & 37.1 & 44.8 & 47.1 \\
\hline & & Experiment 2 & \\
\hline \multicolumn{4}{|l|}{ Cuing** } \\
\hline Cued & 35.6 & 36.2 & 41.2 \\
\hline \multirow[t]{2}{*}{ No Cue } & 37.9 & 44.0 & 44.9 \\
\hline & & Experiment 3 & \\
\hline \multicolumn{4}{|l|}{ Fixation $\dagger$} \\
\hline Valid & 28.7 & 34.2 & 37.2 \\
\hline Neutral & 32.7 & 37.8 & 39.6 \\
\hline Invalid & 40.2 & 44.7 & 46.4 \\
\hline
\end{tabular}

* On cued trials a verbal location cue was supplied, and fixation was shifted to the location of the target letter. **On cued trials a verbal location cue was supplied, but fixation was always centered. $\quad$ tA verbal location cue was never supplied, but fixation varied randomly from trial to trial.

the two types of nonword context $[\mathrm{W}+(\mathrm{min})=71$, $\mathrm{p}>.05]$.

Cued condition. It was also predicted that whenever the location of the target was known in advance of the presentation of the test display, the effects of lexical context would be severely attenuated. The success of this expectation is readily apparent, since the $10 \%$ word advantage in the no-cue condition is reduced to a $3 \%$ advantage when target location is cued in advance. Only 9 of the 14 subjects still show a word advantage in the cued condition and a Wilcoxon test indicates that the word/consonant string differences are not significant $[\mathrm{W}+(\max )=72, \mathrm{p}>.05]$. The small $2.3 \%$ advantage for pseudowords over consonant strings observed in the no-cue condition was reduced by the location cue to $1.6 \%$. Perhaps more meaningful was the fact that cuing reduced the number of subjects showing the pseudoword advantage from 10 to 6.

Dissimilar errors. It was suggested that the frequency of incorrect responses dissimilar to the target provided a rough estimate of how often subjects make an error based on information from a contextletter location rather than the target-letter location. About $23 \%$ of all the trials (58\% of all errors) were errors of this type. Table 2 shows a breakdown of these dissimilar errors for each condition of context type and cuing. Each entry is the percentage of all trials (not the percentage of all errors) on which a dissimilar alternative was reported. The model predicts that the frequency of this type of error should increase under conditions of positional uncertainty, and furthermore, that the magnitude of this increase should depend on the type of context. The results are consistent with these expectations. When the location cue was not provided, the incidence of dissimilar errors increased significantly $[\mathrm{W}+(\mathrm{min})=91$ and 92 , $\mathrm{p}<.01]$ by $5.2 \%$ for pseudowords and $5.6 \%$ for consonant strings. In contrast, the effects of cuing were not significant in the word condition $[\mathrm{W}+(\mathrm{min})=$ $72, \mathrm{p}>.05]$.

In isolation, the above tests provide rather weak support for the conclusion that location cuing differentially affects words and nonwords. In fact, an analysis of variance on the dissimilar error data does not yield a significant Context Type by Cuing interaction $[F(2,26)=1.43, p>.05]$. However, the same pattern of interaction observed in the analysis of dissimilar errors is also obtained in the analysis of highly confusable displays described later.

Similar errors. It was argued that the frequency of incorrect responses similar to the target could be used as an index of how often subjects make an error on the basis of information found in the target-letter location. About $17 \%$ of all the trials $(42 \%$ of all errors) were errors of this type. The model predicts that the frequency of similar errors should not be affected by positional uncertainty. This prediction is supported by nonsignificant Wilcoxon tests comparing the cued and no-cue conditions for each context type ( $p>.05$ in all cases).

Confusability of context letters. The preceding results support the view that the context letters of a word are seen more clearly than those of a nonword. Consequently, under conditions of positional uncertainty, context letters of nonword displays are frequently confused with the distractor targets. This high incidence of errors based on context-letter input produces an overall advantage in the word condition over the nonword conditions. Since cuing reduces the frequency of this type of error in the nonword displays, the results also support the claim that the ben-

Table 2

Mean Percentage of Dissimilar Errors for Each Context Type and the Two Conditions of Cuing

\begin{tabular}{cccc}
\hline & \multicolumn{3}{c}{ Context Type } \\
\cline { 2 - 4 } & Words & Pseudowords & Consonants \\
\hline Cuing* & \multicolumn{3}{c}{ Experiment 1 } \\
Cued & 19.2 & 20.0 & 23.2 \\
No Cue & 22.5 & 25.2 & 28.8 \\
& & Experiment 2 \\
Cuing** & & & \\
Cued & 18.9 & 17.9 & 23.3 \\
No Cue & 22.4 & 24.6 & 25.8 \\
\hline
\end{tabular}

* On cued trials a verbal location cue was supplied, and fixation was shifted to the location of the target letter. **On cued trials a verbal location cue was supplied, but fixation was always centered. 
efits of lexical context can be erased when subjects attend to and consider only the input from the targetletter location.

These conclusions were put to a second test based on the confusability between context letters and the set of distractor targets. Displays with context letters that are very similar to the distractor targets should be extremely error prone under conditions of positional uncertainty. However, performance should improve when a location cue is provided. On the other hand, displays with context letters that are very distinctive from the incorrect targets should rarely be susceptible to errors based on context-letter input. In this condition, the location cue should not be useful even when it is supplied.

The following procedure was used to scale each of the test displays on the likelihood that information from the context-letter locations would lead to an error. For each display, Townsend's (1971, Table 1) alphabetic confusion matrix was used to evaluate the confusability of each of the three context letters to each of the targets. This table presents the proportion of specific letter responses to all letter stimuli. Proportions associated with incorrect responses were assigned positive values, while proportions associated with the correct response were assigned a negative value. The confusion value for a display was defined as the algebraic sum of the individual proportions.

Consider, as an example, the confusion (CON) value computed for the pseudoword CHUP. From Townsend, the proportions of $\mathrm{M}, \mathrm{N}, \mathrm{P}$, and $\mathrm{R}$ responses to the stimulus $C$ are $.00, .00, .03$, and .02 , respectively. This means that if a response was based on the input from the context-letter $\mathrm{C}$, the subject would be slightly more likely to respond correctly with $P$ than with any of the incorrect targets. Assigning the correct response, $\mathrm{P}$, a negative value, the contribution of $\mathrm{C}$ to the CON value for this display becomes: $.00+.00+-.03+.02=-.01$. The proportions of $M, N, P$, and $R$ responses to the stimulus $H$ are $.03, .15, .02$, and .04 . Since an $H$ is very likely to be confused with an $\mathrm{N}$ and somewhat likely to be seen as an $\mathbf{M}$ or $\mathbf{R}$, the context-letter $\mathbf{H}$ has a potentially high source of confusability: $.03+.15+$ $-.02+.04=+.20$. The proportions of $\mathrm{M}, \mathrm{N}, \mathrm{P}$, and $R$ responses to the context-letter $U$ are $.02, .05$, .01 , and .01 . This yields a CON value of +.07 for the contribution of the context-letter $U$. Therefore, the total CON value for the display CHUP is $-.01+$ $.20+.07=+.26$

The 80 displays of each context type were partitioned according to their confusability with the targets. For each context type, the $\mathbf{3 0}$ displays with the highest CON value were compared to the displays with the lowest $C O N$ value. The mean $C O N$ values for the three context types, shown in the first column of Table 3, are reasonably matched, although the
Table 3

Mean Percentage of Errors on the Most and Least Confusable Displays of Each Context Type

\begin{tabular}{lcccccc} 
& $\begin{array}{c}\text { CON } \\
\text { Value }\end{array}$ & \multicolumn{2}{c}{ Experiment 1 } & & \multicolumn{2}{c}{ Experiment 2 } \\
\cline { 3 - 5 } \cline { 5 - 6 } & Cued & No Cue & Cued & No Cue \\
\hline Most & .21 & 44.8 & 42.6 & 36.7 & 42.1 \\
Least & .04 & 29.0 & 32.1 & 34.5 & 35.2 \\
& \multicolumn{5}{c}{ Pseudowords } \\
Most & .21 & 44.8 & 54.8 & 42.3 & 53.0 \\
Least & .05 & 38.3 & 38.8 & 36.2 & 40.5 \\
& & \multicolumn{5}{c}{ Consonants } \\
Most & .23 & 42.9 & 52.9 & 45.2 & 48.8 \\
Least & .02 & 36.0 & 38.8 & 34.3 & 37.4 \\
\hline
\end{tabular}

Note-CON values are the mean values for the 30 displays of each context type that yield the highest (most confusable) and lowest (least confusable) confusion values as computed from Townsend's (1971) confusion matrix and the algorithm described in the text.

consonant strings show a slightly wider range of confusability. The second and third columns show the percentage of errors in the cued and no-cue condition for each combination of context type and level of confusability.

Since errors based on context-letter input are likely to occur only when the context letters are similar to the targets, the perceptual confusion model predicts that the most confusable displays should yield the same pattern of effects that was obtained in the analysis of dissimilar errors. Wilcoxon tests show that the location precue significantly reduced the error rate by $10 \%$ for both pseudowords $[\mathrm{W}+(\mathrm{min})=85$, $\mathrm{p}<.05]$ and consonant strings $[\mathrm{W}+(\min )=80$, $p<.05]$, but had no effect on word displays. This is precisely the pattern of effects obtained in the analysis of dissimilar errors, and further supports the hypothesis that lexical context facilitates the perception of the context letters of words to the extent that they are much less likely to be confused with one of the incorrect targets than are the context letters of nonwords. An analysis of variance on the error rates for the most confusable displays supports the inferred interaction between context type and cuing $[F(2,26)=$ $3.78, \mathrm{p}<.05]$.

Wilcoxon tests comparing the cued and no-cue conditions of the displays with low confusion values are all insignificant $(p>.05)$. This mirrors the null results found in the analysis of similar errors and is consistent with the hypothesis that cuing eliminates errors based on context-letter input, but does not facilitate the extraction of information from the target letter.

\section{EXPERIMENT 2}

It appears that the cuing procedure used in the first experiment effectively erases the effects of lexical 
context. As hypothesized, the benefits of the location precue were nested solely in a reduction of the number of dissimilar errors. An additional test showed that the location precue was most effective when the context letters of a display were highly confusable with the set of distractor targets. We argued that these results are consistent with the view that the primary function of the location cue is to permit the subject to isolate and consider only the visual information from the target-letter location. The purpose of the second experiment was to determine if foveal fixation of the target letter is a necessary condition for segregating the target letter from the surrounding context letters. To this end, the lateral shifting of the fixation point in the cued condition was eliminated. Subjects were informed prior to the start of the experiment that the fixation point would always be centered between the two middle letters of the test display. The apparatus, materials, and procedure were in all other ways the same as in Experiment 1. A different set of 14 students served as subjects.

\section{Results}

An analysis of variance was performed on the error rates treating cuing, context type, and target location as separate factors. The main effects of cuing $[F(1,13)=8.00, p<.05]$, context type $[F(2,26)=$ $6.32, \mathrm{p}<.01]$, and target location $[\mathrm{F}(3,39)=5.62$, $\mathrm{p}<.01]$, and the Cuing by Context Type interaction $[\mathrm{F}(2,26)=3.66, \mathrm{p}<.05]$ were significant. The main effect of target location is very similar to that obtained in the first experiment; displays with the target in the first position averaged about $9 \%$ fewer errors than those with targets in the other positions. Again, target location failed to interact with any other factor, and the data were collapsed across target location in all further analyses.

Effects of location cue. The middle panel of Table 1 shows the average percentage of errors obtained in Experiment 2 for the three context types and the two conditions of cuing.

The cuing effects for pseudowords and words were very comparable to those found in the first experiment. Performance on pseudowords was significantly better in both experiments when a location cue was provided $(6.8 \%$ in Experiment 1 and $7.8 \%$ in Experiment 2), but words showed only nonsignificant benefits (.3\% in Experiment 1 and $2.3 \%$ in Experiment 2).

For the consonant strings, the verbal location cue provided in the second experiment was not as effective as it was in the first experiment, where the verbal cue was combined with a fixation shift to the target location. The cuing effect was large $(7.3 \%)$ and significant in the first experiment, but smaller $(3.7 \%)$ and nonsignificant in Experiment 2 . However, it is not easy to interpret this difference, since a Wilcoxon rank-sum test shows that the magnitude of the cuing effect obtained in Experiment 1 is not significantly greater than that obtained in Experiment $2(S=226$, $\mathrm{p}>.05)$.

No-cue condition. The no-cue condition of Experiment 2 was an exact replication of the no-cue condition of Experiment 1. Accordingly, the same pattern of context effects was expected and obtained. Targets in words were identified $7 \%$ better than targets embedded in consonants. Thirteen of the 14 subjects showed this word advantage, and a Wilcoxon test shows that the word vs. consonant string difference is significant $[\mathrm{W}+(\mathrm{min})=99, \mathrm{p}<.01]$. Again, there was no significant difference between the pseudowords and the consonant strings $[\mathrm{W}+(\mathrm{min})=58$, $p>.05]$.

Cued condition. The ineffectiveness of the location cue for consonant strings permitted the advantage of words over consonants to be carried over into the cued condition $[\mathrm{W}+(\mathrm{min})=84, \mathrm{p}<.05]$. However, the benefits of lexical context were attenuated. Of the 13 subjects showing a word advantage in the no-cue condition, only 9 still showed an advantage in the cued condition. Consistent with the results of the first experiment, the location cue increased performance on the pseudowords to the same level as the words. Thus, targets in pseudowords, like targets in real words, were better recognized than targets embedded in consonant strings $[\mathrm{W}+(\mathrm{min})=85, \mathrm{p}<.05]$.

Dissimilar errors. About $22 \%$ of all the trials $(55 \%$ of all errors) were incorrect responses dissimilar from the target presented. The lower panel of Table 2 shows the breakdown of this type of error for each condition of context type and cuing. It was expected that any benefits of cuing on overall performance would be nested in the frequency of dissimilar errors. The results are consistent with this hypothesis. When the location of targets embedded in pseudowords is cued in advance, the $7.8 \%$ decrease in overall error rate is almost entirely accounted for by the $6.7 \%$ reduction in dissimilar errors. By contrast, cuing reduces the number of dissimilar errors in words and consonant strings by only $3.5 \%$ and $2.5 \%$, respectively. The cuing effect is highly significant for pseudowords $[\mathrm{W}+(\min )=101, \mathrm{p}<.005]$, but not for either of the other context types. This evidence supports the view that a verbal location cue will reduce the number of errors based on context-letter input for pseudowords, but has a rather negligible effect on words or consonants. The analysis of highly confusable displays that is described later supports the same conclusion and attenuates concerns that might otherwise arise from the fact that an analysis of variance on the dissimilar error data does not yield a significant Cuing by Context Type interaction $[F(2,26)$ $=1.58, \mathrm{p}>.05]$.

Similar errors. About $18 \%$ of all the trials $(45 \%$ of 
all errors) produced incorrect responses that were similar to the target presented. As expected, the incidence of similar errors in the three context conditions was not affected by cuing.

Confusability of context-letters. The fourth and fifth columns of Table 3 show the percentages of errors in the cued and no-cue conditions for each combination of context type and level of confusability. Consistent with the results of the first experiment, it is apparent that the highly confusable displays are much more error prone than those with low confusion values. However, the effect of location cuing on consonant strings is markedly different in the two experiments. A location precue reduced the error rate on the most-confusable consonant displays by $10.0 \%$ in the first experiment, but only by $3.6 \%$ in the second. The latter effect is not significant $[\mathrm{W}+(\mathrm{min})=$ $65, p>.05]$. In contrast, the cuing effects on the most-confusable pseudowords were almost identical in the two experiments. The $10.7 \%$ reduction in errors found in the cue condition of the second experiment is highly significant $[\mathrm{W}+(\mathrm{min})=90, \mathrm{p}<.05]$. The effectiveness of the location cue for the pseudowords and its relative ineffectiveness for the consonants is, of course, precisely the pattern of results obtained in the preceding analysis of dissimilar errors. Inspection of the means for word displays suggests that the highly confusable words may also benefit from cuing. However, only 8 of the 14 subjects have fewer errors on the cued trials and a Wilcoxon test indicates a nonsignificant difference [W $+(\max )$ $=78, \mathrm{p}>.05$ ].

Wilcoxon tests comparing the cued and no-cue conditions of the displays with low confusion values are all insignificant ( $p>.05$ in all cases). This replicates the null results found in the analysis of lowconfusion displays in Experiment 1.

Summary of Experiments 1 and 2. The first two experiments present a provocative interaction between the three context types and the two methods of location cuing. Focusing on only the no-cue conditions would lead to the conclusion that words are very special, since a word context produces about a $7 \%$ advantage over either pseudowords or consonant strings. However, differences between pseudowords and consonants can be seen in the cued condition of Experiment 2. A verbal location cue is sufficient to improve performance with a pseudoword context, but not for the consonant case. This would suggest that verbal cues are sufficient to segregate the target from a word-like background, but that the additional assistance of foveal fixation is necessary to segregate a target from an unrelated background of consonant letters. This view is supported by the fact that cuing significantly reduces the incidence of dissimilar errors for both pseudowords and consonants in Experiment 1 , but only for the pseudowords in Experiment 2 .

\section{EXPERIMENT 3}

The pattern of results in the first two experiments differed only when targets were presented in consonant strings. For this type of context, the verbal location cue may be less effective by itself than it is with concomitant shifts in the fixation position. It appears that foveal fixation of the target location may be a necessary condition for completely erasing the benefits of lexical context. We assume that the primary function of foveal fixation is to facilitate a segregation process that, in turn, permits a response to be based only on information about the target. Alternative interpretations could be offered that place primary emphasis on the fact that foveal fixation provides a greater acuity and hence more information about the target letter. The third experiment seeks to reject this class of explanation by showing that the increase in perceptual clarity due to foveal fixation cannot explain the Cuing by Context Type interaction obtained in Experiment 1 . In the third experiment, the fixation conditions of Experiment 1 are reinstated, but verbal location cues are never supplied.

\section{Method}

Three fixation conditions were used. In the neutral condition, the fixation point was perfectly centered. This constituted an exact replication of the no-cue condition of the first two experiments. In the valid condition, the fixation point was laterally shifted to the position of the upcoming target. This replicates the foveal view of the target letter introduced in the cued condition of Experiment 1 , but without the additional benefit of a verbal precue. In the invalid condition, the fixation point was shifted to the mirror-image location of the target; for example, for the word RIFT, the fixation point was aligned under the context-letter $T$ and for the word TREK the fixation was beneath the contextletter $\mathrm{E}$.

The invalid condition was used to insure that subjects could not anticipate the location of the target letter from the apparent location of the fixation point in the preexposure field. To this end, the fixation condition randomly varied from trial to trial. As in the prior experiments, it was emphasized that it was extremely important for subjects to always look directly at the fixation point. However, in this case, subjects were not told about the relationship between fixation position and target location. Postexperimental interviews revealed that none of the 14 subjects became aware of either the mirror-image arrangement used on invalid trials or the fact that one-third of the trials used a valid fixation position. In fact, most of the subjects believed that the fixation position was independent of the target location. Therefore, it is reasonable to assume that the subjective level of uncertainty about target location was the same in this experiment as in the no-cue conditions of the first two experiments.

The 240 different displays were presented in the same random order in each of three experimental sessions. Fixation condition was randomized within sessions. The only restriction was that across the three sessions each display be presented once under each of the three fixation conditions. In all other ways, the apparatus, materials, and procedure were the same as in the prior experiments.

\section{Results}

A large main effect of fixation condition was obtained. Error rates drop from .44 to .37 to .33 for the 
invalid, neutral, and valid conditions, respectively. Wilcoxon tests indicate that an invalid fixation point significantly impairs performance relative to either the neutral or valid fixation positions, $W+=100$ and 105 , respectively $(p<.005)$. However, the effects of neutral and valid fixation positions do not significantly differ from one another.

There is also a strong main effect of context type. Targets embedded in words, pseudowords, and consonant strings yield error rates of $.34, .39$, and .41 , respectively. The effects of word context significantly differ from both nonword contexts $[\mathrm{W}+(\mathrm{min})=99$ and 102 , respectively, $p<.005$ ]; but the advantage of pseudowords over consonants failed to reach significance.

The bottom panel of Table 1 shows the average percentage of errors obtained with the three context types for each of the conditions of fixation. The effects of lexical context are quite consistent across fixation conditions. In all cases, targets embedded in words are recognized better than those in pseudowords, which, in turn, enjoy a slight advantage over consonant strings. Wilcoxon tests show that the magnitude of these differences are not affected by fixation position, indicating that context type and fixation position do not interact. These results render untenable any interpretation of the Cuing by Context Type interaction found in Experiment 1 that is based on changes in the retinal location of the target. The nonsignificant trends are even in the wrong direction. If foveal viewing served to eliminate the context effects in Experiment 1, one would expect the context effects obtained in Experiment 3 to diminish as the target was projected closer to the fovea. But the differences between words and consonant strings actually increase from $6.2 \%$ to $6.9 \%$ to $8.5 \%$ for the invalid, neutral, and valid fixation conditions.

\section{DISCUSSION}

\section{Could Orthographic Redundancy \\ Have Produced Our WSE?}

The perceptual-confusion model assumes that the primary benefits of lexical context accrue from the activation of whole-word units. This hypothesis is supported by the fact that in the no-cue conditions of the present experiments (1) words are superior to both pseudowords and consonant strings and (2) both types of nonwords do not significantly differ from each other.

Massaro (1973; Thompson \& Massaro, 1973) has correctly argued that the benefits of orthographic redundancy can be controlled with predesignated targets. However, this logic applies only to the conditions that Massaro tested, viz, conditions where target location is known in advance of stimulus presentation. Accordingly, under conditions of positional uncertainty, a model based on orthographic constraint would predict that words with high levels of orthographic structure would provide better context than nonwords with lower levels of orthographic structure. It would seem necessary, then, to show that orthographic redundancy can not account for our word advantages.

Among those studies that have used predesignated targets, the present experiments are the first to use pseudowords whose orthographic structure is comparable to word displays. Table 4 shows the amount of orthographic structure for each of the context types used in the present experiments as measured by four commonly used techniques. The first column shows the average bigram frequency for each display type based on the Underwood and Schulz (1960) norms. The second and third columns show the average position-specific bigrams and position-specific single-letter frequencies based on the Mayzner and Tresselt (1965) norms. The last column is the average rating assigned by 69 introductory psychology students who were asked to rate each display on a 10point scale in terms of how word-like it appeared. Inspection of Table 4 clearly shows that the orthographic structure of the pseudowords is nearly equal to that of the words, with both of these context types having considerably more structure than the consonant strings. This strongly suggests that the word advantages obtained in these experiments are primarily due to lexical access rather than inferences based on orthographic knowledge.

Before rejecting completely the hypothesis that orthographic structure contributes to the context effects observed in the present experiments, we decided to try a series of post hoc comparisons between the most and least structured displays of each type. For each measure and context type, the 30 displays with the highest and lowest levels of orthographic structure and the average percentage of errors for the high and low displays of each type collapsed over the nocue conditions of Experiments 1 and 2.

Although it may be possible to map an intriguing story onto the exceedingly complex pattern of results shown in Table 5, it seems prudent to highlight only a few observations. First, only the subjective ratings

Table 4

Mean Value of Orthographic Structure for Words, Pseudowords, and Consonants

\begin{tabular}{lrrrc}
\hline & \multicolumn{3}{c}{ Measure of Orthographic Structure } \\
\cline { 2 - 5 } & BF & $\begin{array}{c}\text { Position- } \\
\text { Specific BF }\end{array}$ & F & $\begin{array}{c}\text { Subjective } \\
\text { Rating }\end{array}$ \\
\hline Words & 2926 & 132 & 1624 & 7.14 \\
Pseudowords & 2818 & 111 & 1446 & 5.14 \\
Consonants & 638 & 22 & 941 & 1.40 \\
\hline
\end{tabular}

Note-BF = bigram frequency; $F=$ single-letter frequency. 
Table 5

Mean Percentage of Errors for the 30 Words, Pseudowords, and Consonants Having the Most and Least Amounts of Orthographic Structure

\begin{tabular}{|c|c|c|c|c|}
\hline \multirow[b]{2}{*}{, } & \multicolumn{4}{|c|}{ Measure of Orthographic Structure } \\
\hline & $\begin{array}{l}\text { Bigram } \\
\text { Frequency }\end{array}$ & $\begin{array}{l}\text { Position-Specific } \\
\text { Bigram Frequency }\end{array}$ & $\begin{array}{l}\text { Single-Letter } \\
\text { Frequency }\end{array}$ & $\begin{array}{c}\text { Subjective } \\
\text { Rating }\end{array}$ \\
\hline & \multicolumn{4}{|c|}{ Words } \\
\hline \multirow[t]{2}{*}{$\begin{array}{l}\text { Most } \\
\text { Least }\end{array}$} & $\begin{array}{ll}35.2 * & (4642) \\
39.0 & (1450)\end{array}$ & $\begin{array}{rr}38.7 & (273) \\
37.0 & (22)\end{array}$ & $\begin{array}{l}39.3(2294) \\
36.8 \quad(971)\end{array}$ & $\begin{array}{ll}35.8^{*} & (7.91) \\
40.1 & (6.26)\end{array}$ \\
\hline & \multicolumn{4}{|c|}{ Pseudowords } \\
\hline \multirow[t]{2}{*}{$\begin{array}{l}\text { Most } \\
\text { Least }\end{array}$} & $\begin{array}{ll}44.4 & (4539) \\
44.5 & (1293)\end{array}$ & $\begin{array}{l}48.2 \div(231) \\
39.5 \quad(17)\end{array}$ & $\begin{array}{l}50.8 \dagger(2001) \\
41.0 \quad(920)\end{array}$ & $\begin{array}{l}40.2^{* *}(6.32) \\
46.4\end{array}$ \\
\hline & \multicolumn{4}{|c|}{ Consonants } \\
\hline $\begin{array}{l}\text { Most } \\
\text { Least }\end{array}$ & $\begin{array}{l}39.5 \dagger(1382) \\
50.2 \quad(\quad 83)\end{array}$ & 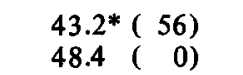 & $\begin{array}{l}48.3^{*}(1491) \\
45.1 \quad(454)\end{array}$ & $\begin{array}{ll}42.0 \dagger & (2.02) \\
49.6 & (.82)\end{array}$ \\
\hline
\end{tabular}

Note-Numbers in parentheses indicate the mean value of structure for each set of 30 displays. The p values signify significant differences, as determined by Wilcoxon signed-ranks tests, between individual pairs of displays with the highest (most structure) and lowest (least structure) values of the indicated measure. $\quad{ }^{*} p<.05 . \quad{ }^{* *} p<.01 . \quad t p<.005$.

show lower error rates for the most word-like displays of all three context types. Second, two of the largest differences observed, those for pseudowords based on position-specific single-letter frequencies and position-specific bigram frequencies, show error rates about $10 \%$ higher on the displays with the greatest, not the least, amount of orthographic structure. Third, it is the consonant strings that show the most consistent effects of orthographic structure; only the position-specific single-letter frequencies fail to result in lower error rates for the highly structured consonant displays. This last observation may suggest that orthographic structure plays a modest role in letter recognition when extremely low levels of structure are compared to somewhat higher levels. However, the advantage of words over pseudowords, and the equivalence of pseudowords and consonants, can not be explained on the basis of any simple interpretation of these findings.

Although the orthographic structures of the words and pseudowords used in the present experiments were quite comparable in terms of the frequencybased measures specified in Table 4, they may differ considerably in terms of rule-governed descriptions of orthographic structure. For example, many of our pseudowords would be irregular according to the rules used by Massaro, Venezky, and Taylor (1979). Should others want to test their own ideas against the data, we have reproduced the results for each of the 240 stimulus items in Appendix A.

\section{Is Our Cuing Effect Simply the Abandonment of Word Processing?}

Johnston and McClelland (1974) have suggested that subject-controlled strategies are critical to obtaining the WSE. In their first experiment, all of the stimuli were four-letter words, whereas in the second experiment they were unpronounceable quadrigrams. In each experiment, the subject's strategy was directly manipulated through instructions. For half of the trial blocks, subjects were instructed to fixate the middle of the display and to try to see the whole stimulus. On the remaining trials, subjects were told the position of the target letter and were further instructed to fixate that position and try to see only the letter that appeared there. Instructing subjects to pay attention to the whole stimulus, rather than to just the target letter, produced better performance with word stimuli (Experiment 1) but worse performance with unrelated letters (Experiment 2). Combining the data from both experiments leads to the inference that the WSE occurs when subjects allocate their attention across all letters of the display, but that nonwords produce better performance when subjects fixate and attend to only the target position.

The strategy notion provides a second reason for expecting a Cuing by Context Type interaction. If subjects abandon a strategy of deliberately attending to the whole display when target location is known, then the cuing effect should be caused primarily by a decrease in performance on words, with little change expected for nonword displays. In contrast, if the main role of the location cue is to permit subjects to consider only the input from the target location and avoid confusions arising from the context letters, then the cue should primarily lead to an increase in performance on nonword trials with little change occurring on word trials. This follows because the perceptual-confusion model predicts that nonwords should be much more susceptible to these confusion errors than words. In the present experiments, providing a location cue neither helps nor hurts performance on words, but leads to significant increases in nonword performance. This would suggest that 
most of the Cuing by Context Type interaction results from the large benefits of positional certainty for nonwords, rather than from costs in the processing of words that might occur when attention is shifted away from the whole display and toward a single letter.

Although the obtained cuing effects seem to fit the predictions of the perceptual-confusion model without the need for additional explanations, it is difficult to counter the argument that subject-controlled strategies may have played a small role in the present experiments. Specifically, one possibility is that the location cue had no effect on words because of a trade-off between the detrimental effects of abandoning a word-processing strategy and the benefits of selectively attending to the target-location input. However, it is unlikely that our subjects would deliberately attempt to process all displays as whole words whenever there was positional uncertainty because only one-third of the randomly presented displays were words. Accordingly, we favor the alternative hypothesis, that the benefits of lexical context shown in our no-cue condition reflect the obligatory activation of the lexicon rather than the controlled processing of all displays as whole words.

\section{Can a WSE Occur When Target Location is Known and Fixated?}

A recent study by Purcell, Stanovich, and Spector (1978) appears, at first, to blatantly contradict the effects of positional uncertainty reported in the present studies and to seriously weaken the model advanced. In two experiments, Purcell et al. report large word advantages even though predesignated targets are used, there is complete certainty about target position, the subject is fixating the position of the target letter, and only two context frames are used. These experiments used the same four words (APE, ARE, ACE, AGE) and nonwords (VPH, VRH, VCH, VGH) as Massaro (1973), but employed a much smaller visual angle, $.53 \mathrm{deg}$ rather than $3.33 \mathrm{deg}$. Purcell et al. conclude that the visual angle of the stimulus display is a crucial factor in determining the presence of word advantages, since word advantages are likely to occur only with small visual angles.

Purcell et al. conclude that supraletter features can be automatically extracted only when the component letters are in or near the fovea. An alternative explanation for the word advantages observed with these small stimuli is differential lateral masking. The A_E frame may interfere less with the perception of the target letter than the $\mathrm{V}_{-} \mathrm{H}$ frame. If such differential lateral masking existed, the effects would be more apparent when the letters were close together than when they were farther apart. This follows since separation is one of the important determinants of the magnitude of lateral masking (Bouma, 1970). This would explain the absence of word advantages in both Massaro's original report and in Experiment 3 of Purcell et al., since in both cases the separation between adjacent letters was about $.7 \mathrm{deg}$. In contrast to this rather large interletter spacing, the context letters of the small displays used in their first two experiments are nearly touching the target letter.

The implications for a lateral masking reinterpretation of these results are obvious, since this explanation rests simply on the difference in visual structure between the $A_{-} E$ and $V_{-} H_{\text {frames. It }}$ becomes only a fortuitous coincidence that the target letters formed words in the context of the less effective mask. With this reinterpretation in mind, we have recently conducted and reported (Paap \& Newsome, 1980) a series of experiments that: replicate the results of Purcell et al.'s first experiment under similar conditions (Experiment 1), show that these word advantages do not require a patterned mask as do those observed by others (Experiment 2), show that the $\mathrm{V}_{-} \mathrm{H}$ context can laterally mask a set of target digits more effectively than the A_E context (Experiment 3), and finally that the word advantages found under the conditions imposed by Purcell et al. with the $A_{-} E$ and $V_{-} H_{\text {contexts do }}$ not generalize to another set of materials (Experiments 4 and 5).

\section{Conclusions}

This study investigated the benefits of lexical context in the predesignated targets paradigm in conditions where target location was either known or unknown. Under conditions of positional uncertainty, words provided a more effective context than either pseudowords or consonant strings. This was particularly true for displays that contained context letters that were highly confusable with the incorrect targets. In contrast, when a location precue was accompanied by foveal fixation of the target location, these word advantages were severely attenuated.

The obtained pattern of results was predicted by the perceptual confusion model outlined in the introduction. This model assumes that the perceptual recognition process involves a continuous synthesis of the features that are detected and held in a preperceptual visual store. The perceptual synthesis relies on distinctive feature lists that correspond both to individual letters and familiar words. We assume that the matching of the input features to letter and word candidates occurs concurrently and that some words can be synthesized on the basis of fewer infraletter features than would be necessary to activate each of the constituent letter units. Since the same set of detected features can lead to the formation of a correctly perceived word, but only partial resolu- 
tion of the same letters presented in isolation, lexical context should often produce an advantage of words over nonwords.

In the postcue paradigm, word matches are likely to lead to an overall increase in the number of correctly synthesized letters, that is, lexical context should benefit both the target letter and the set of context letters. However, when the targets are predesignated, the benefits of a word match should be greater for context letters than for the target letter. This follows from the assumption that the criterial number of features will be lowered for the letter units corresponding to the target set. This bias favoring the target letters should increase the likelihood that the input from the target letter will be synthesized as itself, but should also increase the likelihood that input from a context letter will be synthesized as one of the three incorrect targets. Thus, in the predesignated targets paradigm, discrepancies between matches at the level of individual letter units and matches at the word level should involve context letters much more frequently than target letters. Accordingly, the benefits of lexical context can be seen only when target location is unknown and it is possible for context letters to be confused as potential targets. It also follows that a location precue can effectively eliminate word advantages to the extent that subjects can focus on the target input and ignore the context letters.

It should not be presumed that word advantages should occur whenever there is uncertainty about the target location. For example, the benefits of lexical access on context-letter recognition are likely to be most pronounced when a wide variety of words and nonwords are used and there is considerable uncertainty about the identity of the context letters. However, the facilitation should be weak, or even absent, if a restricted set of context frames are used repeatedly. In these cases, the context letters can be resolved on the basis of partial information and should not be seen as potential targets.

We have already shown that, under conditions of positional uncertainty, the magnitude of the WSE will increase with the level of confusability between the context letters and the incorrect target alternatives. Similar logic would predict that the magnitude of the word advantages should increase with the number of letters in the target set. If four-letter displays contain a single target from a master set of only two targets, then there are only three potential confusion errors, that is, each of the three context letters could be misperceived as the single incorrect target alternative. In contrast, when there are four letters in the master set of targets, there are now nine potential confusion errors. This is because each of the three context letters must be compared to each of the three incorrect targets and any of the nine comparisons could lead to the synthesis of an incorrect target. Although the relationship between the size of word advantages and the number of targets remains to be systematically explored, it may be the cause of some failures to find word advantages with predesignated targets and positional uncertainty. Carr, Lehmkuhle, Kottas, Astor-Stetson, and Arnold (1976) and the present studies used four target alternatives and obtained word advantages. Bjork and Estes (1973), Estes (1975), and Estes, Bjork, and Skaar (1974) used only two alternatives and found no effects of context. The only exception to this pattern was reported by Spector and Purcell (1977), who obtained significant word advantages with only two targets.

The perceptual-confusion model is more a product of others' inspiration than our ingenuity. The view that a continuous perceptual synthesis can be guided by linguistic knowledge is drawn directly from Massaro (1975). The perceptual-confusion model differs from Massaro's primarily in our emphasis on the role of lexical, rather than orthographic, constraint. Both types of linguistic context may be needed to account for context effects in the postcue procedure, and orthographic redundancy may become important in the predesignated targets paradigm when longer displays are used (cf. Massaro, Venezky, \& Taylor, 1979). The idea that distractors may sometimes be confused with targets is, of course, not a new idea either. For example, Gardner (1973) and Gummerman (1973) have shown that this type of error may be involved in many investigations of selective perception. Furthermore, Estes (1975) has considered the role of positional uncertainty in both the predesignated and the postcued targets paradigms. Estes suggests that, when the alternatives are postcued, words provide better context than nonwords, since familiar patterns supply positional information that enhances the chances that input arising from the target will be attributed to its actual position in the display. On the basis of his experiments using predesignated targets, Estes concludes that linguistic context does not facilitate perceptual recognition, but that the advantages of single letters over both words and nonwords may be due to the fact that confusions can occur only in multiletter displays. Our contribution, we believe, is the evidence that supports the view that the perceptual recognition of words involves activation of the lexicon, and that lexical access will interact with confusability and positional uncertainty to determine the magnitude of the WSE.

\section{REFERENCE NOTES}

1. Paap, K. R., Newsome, S. L., \& Rudy, K. The locus of contextual and selective attention effects in letter recognition. Paper presented at the Rocky Mountain Psychological Association, Denver, April 1978. 


\section{REFERENCES}

BJork, E. L., \& Estrs, W. K. Letter identification in relation to linguistic context and masking conditions. Memory \& Cognition. 1973, 1, 217-223.

Bouma, H. Interaction effects in parafoveal letter recognition. Nature, 1970, 226, 177-178.

Bradley, J. V. Probability; decision; statistics. Englewood Cliffs, N.J: Prentice-Hall, 1976.

Carr, T. C., Lehmkuhle. S. W., Kottas, B., Astor-Stetson, E. C., \& ARNOLD, D. Target position and practice in the identification of letters in varying context: A word superiority effect. Perception \& Psychophysics, 1976, 19, 412-416.

Estes, W. K. The locus of inferential and perceptual processes in letter identification. Journal of Experimental Psychology: General, 1975, 104, 122-145.

Estes, W. K., Bлork, E. L.. \& Skaar, E. Detection of single letters and letters in words with changing versus unchanging mask characters. Bulletin of the Psychonomic Society, 1974, 3, 201-203

Garuner, G. T. Evidence for independent parallel channels in tachistoscopic perception. Cognitive Psvchology, 1973, 4, $130-155$.

Gummerman, K. A model of selective perception: The effect of presenting alternatives before or after the stimulus. Bulletin of the Psychonomic Society, 1973, 2, 365-367.

Johnston, J. C., \& McCifilland, J. L. Perception of letters in words: Seek not and ye shall find. Science, 1974, 184, 1192-1194.

Massaro, D. W. Perception of letters, words, and nonwords. Journal of Experimental Psychology, 1973, 100, 349-353.

Massaro, D. W. (ED.), Understanding language: An information processing analysis of speech perception, reading, and psycholinguistics. New York: Academic Press, 1975.

Massaro, D. W., Venezky. R. L., \& Taylor, G. A. Orthographic regularity, positional frequency, and visual processing of letter strings. Journal of Experimental Psychology: General, 1979, 108, 107-124.

Mayzner, M. S., \& Tresselt, M. E. Tables of single-letter and digram frequency counts for various word-length and letterposition combinations. Psychonomic Monograph Supplements, $1965,1,13-32$.

PaAP, K. R., \& Newsome, S. L. Do small visual angles produce a word superiority effect or differential lateral masking? Memory \& Cognition, 1980, 8, 1-14.

Purceil, D. G., Stanovich, K. E., \& Spector, A. Visual angle and the word superiority effect. Memory \& Cognition, 1978, 6, 3-8.

REICHer, G. M. Perceptual recognition as a function of the meaningfulness of the material. Journal of Experimental Psychology, 1969, 81, 275-280.

Sмiтн, F. Understanding reading. New York: Holt, Rinehart, and Winston, 1971.

Spector, A., \& Purcell, D. G. The word-superiority effect: A comparison between restricted and unrestricted alternative sets. Perception \& Psychophysics, 1977, 21, 323-328.

Trom pson, M. C., \& Massaro, D. W. Visual information redundancy in reading. Journal of Experimental Psychology, 1973, 98, 49-54.

ThORNDIKE, E. L., \& LoRge, I. The teacher's word book of 30,000 words. New York: Teachers College, Columbia University, 1944.

Townsend, J. T. Theoretical analysis of an alphabetic confusion matrix. Perception \& Psychophysics, 1971, 9, 40-49.

UNDE RWOOD, B. J., \& Schulz, R. W. Meaningfulness and verbal learning. Philadelphia: Lippincott, 1960.

\section{NOTE}

1. Nonparametric statistics were reported in the results of this series of experiments because we feel that they are more appropriate than the more commonly used parametric tests. The data were also analyzed using the analysis of variance and planned linear contrasts on the comparisons of interest. Although the level of significance occasionally fluctuated, the choice of the distributionfree tests never led to a conclusion that would have been different from one based on parametric tests.

Appendix

Words, Pseudowords, and Consonant Strings Used in the Present Experiments

\begin{tabular}{|c|c|c|c|c|c|c|c|c|c|c|c|c|c|c|}
\hline \multirow[b]{2}{*}{ Word } & \multicolumn{2}{|c|}{ Experiment 1} & \multicolumn{2}{|c|}{ Experiment 2} & \multirow{2}{*}{$\begin{array}{c}\text { Pseudo- } \\
\text { word }\end{array}$} & \multicolumn{2}{|c|}{ Experiment 1} & \multicolumn{2}{|c|}{ Experiment 2} & \multirow{2}{*}{$\begin{array}{c}\text { Conso- } \\
\text { nant }\end{array}$} & \multicolumn{2}{|c|}{ Experiment 1} & \multicolumn{2}{|c|}{ Experiment 2} \\
\hline & $\mathrm{C}$ & $\mathbf{N}$ & $\mathrm{C}$ & $\mathbf{N}$ & & $\mathrm{C}$ & $\mathbf{N}$ & $\mathrm{C}$ & $\mathbf{N}$ & & $\mathrm{C}$ & $\mathbf{N}$ & $\mathrm{C}$ & $\mathbf{N}$ \\
\hline PEAK & 3 & 4 & 5 & 5 & POIK & 1 & 3 & 0 & 4 & PLZK & 4 & 5 & 1 & 2 \\
\hline PAVE & 4 & 2 & 0 & 3 & POVU & 0 & 2 & 1 & 1 & PHVK & 5 & 4 & 2 & 4 \\
\hline PLOD & 2 & 2 & 1 & 2 & PLID & 3 & 6 & 4 & 3 & PLVD & 0 & 3 & 1 & 3 \\
\hline PITH & 6 & 3 & 2 & 1 & POTH & 4 & 6 & 2 & 3 & PQTH & 3 & 8 & 4 & 3 \\
\hline POSY & 3 & 3 & 2 & 5 & PISU & 4 & 3 & 2 & 2 & PDSC & 2 & 4 & 1 & 6 \\
\hline RUST & 5 & 2 & 2 & 5 & ROST & 4 & 5 & 3 & 1 & RGST & 4 & 6 & 8 & 3 \\
\hline RIOT & 6 & 2 & 4 & 6 & REIT & 3 & 4 & 4 & 6 & RKHT & 4 & 3 & 6 & 7 \\
\hline RIFT & 0 & 5 & 5 & 4 & RUFT & 6 & 4 & 2 & 3 & $\mathrm{RBFT}$ & 1 & 8 & 4 & 7 \\
\hline RAZE & 3 & 4 & 4 & 5 & REZO & 6 & 7 & 5 & 8 & RTZD & 2 & 6 & 5 & 5 \\
\hline RUSE & 2 & 0 & 0 & 3 & RASU & 5 & 5 & 3 & 5 & RQSW & 4 & 4 & 5 & 5 \\
\hline NEAT & 3 & 1 & 3 & 5 & NIAT & 1 & 3 & 3 & 7 & NSHT & 3 & 8 & 7 & 5 \\
\hline NIGH & 5 & 4 & 7 & 9 & NAGH & 4 & 9 & 4 & 5 & NZGH & 3 & 2 & 1 & 6 \\
\hline NICK & 5 & 6 & 4 & 2 & NOCK & 1 & 9 & 4 & 4 & NFCK & 2 & 7 & 10 & 9 \\
\hline NAVE & 5 & 7 & 6 & 8 & NOVU & 3 & 5 & 6 & 3 & NDVB & 5 & 5 & 4 & 6 \\
\hline NUDE & 3 & 6 & 2 & 5 & NADO & 3 & 6 & 4 & 4 & NTDX & 5 & 8 & 4 & 8 \\
\hline MODE & 3 & 7 & 7 & 5 & MADU & 8 & 8 & 2 & 7 & MTDV & 3 & 7 & 5 & 8 \\
\hline MOLE & 5 & 8 & 6 & 7 & MALU & 6 & 10 & 7 & 14 & MQLZ & 9 & 7 & 5 & 4 \\
\hline MUCK & 8 & 9 & 8 & 5 & MECK & 7 & 6 & 7 & 8 & MDCK & 4 & 9 & 7 & 6 \\
\hline MITE & 6 & 7 & 7 & 8 & MATO & 8 & 11 & 7 & 5 & MCTQ & 6 & 7 & 10 & 8 \\
\hline MESA & 6 & 8 & 4 & 8 & MISO & 4 & 7 & 7 & 8 & MTSC & 4 & 3 & 2 & 2 \\
\hline SPED & 1 & 4 & 5 & 3 & SPID & 7 & 4 & 6 & 6 & SPFD & 5 & 1 & 5 & 3 \\
\hline SPIT & 7 & 6 & 8 & 8 & SPUT & 2 & 6 & 3 & 4 & SPLT & 5 & 5 & 5 & 4 \\
\hline
\end{tabular}


Appendix Continued

\begin{tabular}{|c|c|c|c|c|c|c|c|c|c|c|c|c|c|c|}
\hline \multirow[b]{2}{*}{ Word } & \multicolumn{2}{|c|}{ Experiment 1} & \multicolumn{2}{|c|}{ Experiment 2} & \multirow{2}{*}{$\begin{array}{c}\text { Pseudo- } \\
\text { word }\end{array}$} & \multicolumn{2}{|c|}{ Experiment 1} & \multicolumn{2}{|c|}{ Experiment 2} & \multirow{2}{*}{$\begin{array}{c}\text { Conso- } \\
\text { nant }\end{array}$} & \multicolumn{2}{|c|}{ Experiment 1} & \multicolumn{2}{|c|}{ Experiment 2} \\
\hline & $\mathrm{C}$ & $\mathrm{N}$ & $\mathrm{C}$ & $\mathbf{N}$ & & $\mathrm{C}$ & $\mathrm{N}$ & $\mathrm{C}$ & $\mathrm{N}$ & & $\mathrm{C}$ & $\mathrm{N}$ & $\mathrm{C}$ & $\mathrm{N}$ \\
\hline EPIC & 4 & 3 & 4 & 3 & APUC & 7 & 6 & 5 & 4 & LPBC & 7 & 5 & 7 & 5 \\
\hline APEX & 4 & 6 & 4 & 5 & IPAX & 8 & 7 & 9 & 9 & WPSX & 5 & 11 & 7 & 11 \\
\hline OPUS & 5 & 3 & 2 & 4 & EPAS & 5 & 6 & 7 & 5 & LPVS & 10 & 2 & 5 & 5 \\
\hline DRUG & 8 & 6 & 4 & 3 & DRIG & 3 & 5 & 3 & 4 & DRHG & 5 & 7 & 4 & 8 \\
\hline BRED & 1 & 5 & 5 & 5 & BROD & 9 & 4 & 7 & 5 & $\mathrm{BRCD}$ & 5 & 6 & 6 & 8 \\
\hline GRIT & 5 & 3 & 7 & 3 & GRAT & 6 & 2 & 3 & 7 & GRXT & 4 & 5 & 3 & 8 \\
\hline TROW & 4 & 9 & 2 & 7 & TRUW & 4 & 6 & 6 & 7 & TRCW & 5 & 7 & 10 & 7 \\
\hline TREK & 4 & 5 & 6 & 5 & TROK & 2 & 3 & 2 & 5 & TRDK & 4 & 3 & 2 & 4 \\
\hline KNOT & 4 & 3 & 2 & 6 & KNET & 4 & 7 & 2 & 8 & KNWT & 5 & 8 & 6 & 8 \\
\hline GNAW & 3 & 4 & 3 & 6 & GNIW & 7 & 4 & 6 & 7 & GNZW & 5 & 6 & 7 & 6 \\
\hline UNDO & 4 & 4 & 5 & 5 & ENDA & 4 & 7 & 1 & 5 & FNDK & 8 & 7 & 6 & 8 \\
\hline SNUB & 5 & 3 & 4 & 6 & SNIB & 4 & 7 & 3 & 10 & SNKB & 2 & 7 & 5 & 8 \\
\hline SNAG & 3 & 2 & 2 & 6 & SNOG & 6 & 6 & 9 & 6 & SNDG & 6 & 5 & 4 & 5 \\
\hline AMID & 10 & 6 & 7 & 9 & EMOD & 4 & 10 & 6 & 10 & CMBD & 6 & 7 & 7 & 11 \\
\hline OMIT & 5 & 9 & 9 & 4 & AMUT & 11 & 11 & 8 & 7 & WMLT & 10 & 11 & 9 & 5 \\
\hline EMIT & 9 & 7 & 9 & 5 & AMET & 9 & 11 & 7 & 7 & DMKT & 7 & 10 & 9 & 9 \\
\hline SMUG & 8 & 9 & 8 & 7 & SMIG & 6 & 7 & 3 & 8 & SMCG & 5 & 12 & 6 & 8 \\
\hline SMUT & 8 & 7 & 5 & 8 & SMAT & 6 & 6 & 7 & 8 & SMVT & 9 & 10 & 9 & 6 \\
\hline WEPT & 7 & 6 & 8 & 9 & WAPT & 4 & 10 & 8 & 8 & WCPT & 7 & 9 & 12 & 12 \\
\hline COPE & 3 & 4 & 2 & 1 & CIPA & 6 & 5 & 4 & 4 & CFPV & 7 & 8 & 6 & 6 \\
\hline GAPE & 4 & 4 & 5 & 4 & GOPA & 3 & 6 & 2 & 3 & GDPT & 6 & 7 & 7 & 1 \\
\hline DUPE & 8 & 4 & 5 & 7 & DIPU & 3 & 5 & 3 & 3 & DXPQ & 1 & 4 & 2 & 4 \\
\hline LOPE & 8 & 4 & 3 & 2 & LAPU & 3 & 5 & 4 & 4 & LFPC & 5 & 5 & 5 & 5 \\
\hline HERS & 3 & 6 & 2 & 5 & HORS & 7 & 3 & 5 & 7 & HKRS & 7 & 7 & 7 & 5 \\
\hline CURB & 6 & 5 & 4 & 4 & CORB & 8 & 4 & 7 & 5 & CVRB & 5 & 10 & 4 & 7 \\
\hline WIRY & 3 & 5 & 7 & 8 & WARO & 8 & 9 & 5 & 6 & WLRD & 9 & 6 & 8 & 6 \\
\hline CURD & 5 & 3 & 3 & 8 & CERD & 9 & 6 & 7 & 6 & CTRD & 6 & 4 & 6 & 2 \\
\hline FURL & 6 & 4 & 8 & 0 & FIRL & 4 & 4 & 6 & 5 & FXRL & 7 & 6 & 10 & 7 \\
\hline LINK & 4 & 7 & 3 & 5 & LENK & 1 & 9 & 4 & 8 & LDNK & 6 & 7 & 5 & 8 \\
\hline BANG & 5 & 2 & 2 & 4 & BUNG & 2 & 3 & 5 & 2 & BWNG & 5 & 5 & 5 & 4 \\
\hline WAND & 2 & 2 & 8 & 6 & WUND & 1 & 9 & 2 & 8 & WLND & 5 & 6 & 4 & 7 \\
\hline ZONE & 3 & 4 & 6 & 4 & ZENU & 6 & 7 & 5 & 7 & ZFNJ & 7 & 4 & 6 & 7 \\
\hline HUNK & 4 & 5 & 6 & 9 & HINK & 5 & 7 & 4 & 5 & HVNX & 7 & 5 & 9 & 5 \\
\hline TAME & 5 & 5 & 6 & 5 & TEMA & 10 & 9 & 4 & 6 & TXMW & 5 & 8 & 10 & 8 \\
\hline DOME & 9 & 11 & 5 & 7 & DIMU & 6 & 6 & 8 & 8 & DCMJ & 11 & 8 & 6 & 8 \\
\hline ALMS & 10 & 7 & 7 & 5 & ULMS & 6 & 8 & 7 & 9 & QLMS & 10 & 10 & 7 & 8 \\
\hline WOMB & 8 & 9 & 5 & 4 & WEMB & 4 & 7 & 9 & 11 & WXMB & 8 & 12 & 7 & 7 \\
\hline COMA & 7 & 7 & 5 & 7 & CAMI & 9 & 8 & 3 & 5 & $\mathrm{CZMH}$ & 7 & 12 & 11 & 11 \\
\hline CLAP & 2 & 3 & 4 & 3 & CLEP & 6 & 0 & 1 & 2 & CLFP & 4 & 4 & 1 & 3 \\
\hline CHAP & 5 & 2 & 3 & 3 & CHUP & 7 & 2 & 2 & 3 & CHTP & 4 & 5 & 4 & 2 \\
\hline FLOP & 5 & 3 & 4 & 6 & FLUP & 1 & 3 & 5 & 4 & FLDP & 7 & 4 & 2 & 1 \\
\hline SWAP & 6 & 5 & 4 & 5 & SWIP & 7 & 3 & 6 & 7 & SWHP & 6 & 4 & 3 & 8 \\
\hline QUIP & 6 & 5 & 5 & 8 & QUEP & 8 & 5 & 3 & 5 & QBZP & 3 & 7 & 2 & 4 \\
\hline HEIR & 3 & 6 & 6 & 6 & HEUR & 4 & 7 & 4 & 7 & HQBR & 6 & 8 & 2 & 10 \\
\hline SOUR & 5 & 3 & 5 & 5 & SUER & 6 & 7 & 8 & 3 & SHLR & 8 & 4 & 3 & 7 \\
\hline LAIR & 6 & 4 & 6 & 3 & LOAR & 6 & 4 & 8 & 6 & LGBR & 4 & 4 & 6 & 6 \\
\hline USER & 7 & 4 & 3 & 3 & ISUR & 6 & 5 & 0 & 5 & CSXR & 8 & 11 & 4 & 4 \\
\hline WEIR & 2 & 7 & 7 & 5 & WUER & 6 & 9 & 8 & 11 & WBFR & 4 & 10 & 7 & 5 \\
\hline TWIN & 3 & 8 & 8 & 4 & TWEN & 3 & 6 & 5 & 7 & TWQN & 6 & 6 & 6 & 8 \\
\hline YAWN & 4 & 5 & 6 & 6 & YUWN & 7 & 5 & 7 & 8 & YHWN & 7 & 6 & 7 & 7 \\
\hline SHIN & 3 & 8 & 4 & 2 & SHEN & 7 & 8 & 5 & 9 & SHWN & 7 & 7 & 6 & 10 \\
\hline HEWN & 4 & 8 & 7 & 7 & HAWN & 10 & 9 & 5 & 9 & HXWN & 4 & 8 & 4 & 7 \\
\hline WAIN & 8 & 6 & 6 & 6 & WUEN & 6 & 8 & 8 & 10 & WHVN & 6 & 7 & 10 & 6 \\
\hline ITEM & 9 & 6 & 5 & 5 & ETAM & 9 & 11 & 11 & 9 & HTJM & 9 & 9 & 8 & 8 \\
\hline SLIM & 8 & 9 & 8 & 9 & SLOM & 8 & 6 & 11 & 8 & SLJM & 9 & 6 & 9 & 7 \\
\hline WHIM & 13 & 10 & 10 & 8 & WHUM & 10 & 11 & 7 & 8 & WHBM & 9 & 8 & 11 & 11 \\
\hline SCUM & 9 & 6 & 7 & 8 & SCOM & 5 & 7 & 11 & 9 & SCXM & 8 & 9 & 8 & 12 \\
\hline HOLM & 9 & 10 & 11 & 9 & HILM & 6 & 12 & 8 & 10 & HLVM & 5 & 9 & 8 & 5 \\
\hline
\end{tabular}

Note-Also shown are the total number of errors, out of a possible 14 , for each item in the cued (C) and no-cue (N) conditions of Experiments 1 and 2. 